\title{
HABITAT UTILIZATION BY ASIATIC WILD ELEPHANT (ELEPHUS MAXIMUS) IN PARSA WILDLIFE RESERVE, NEPAL
}

\author{
B.R. Yadav ${ }^{1}$, I.C. Dutta ${ }^{1}$, M.K. Chalise ${ }^{*}$, Christy Williums and B.K. Sharma** \\ ${ }^{1}$ Institute of Forestry, Tribhuvan University, Pokhara, Nepal \\ *Central Department of Zoology, Tribhuvan University, Kathmandu, Nepal \\ ${ }^{* *}$ Green Governance Nepal, Kathmandu, Nepal \\ Email: yadav.baburam@gmail.com
}

\begin{abstract}
During the habitat survey, elephants foot prints, dungs and dirct observations were performed along each sampling grid. GPS points of elephant's presence were recorded. Aiming to provide quantitative analysis of habitats, circular quadrats of $10 \mathrm{~m}, 2 \mathrm{~m}$ and $1 \mathrm{~m}$ radius were used to collect information about trees, shrubs and herbs. Importance values of each species of trees were analyzed by adding relative frequency, relative density and relative dominance. Name of each forest type was determined from the sequencial order of the large Importance Values (IV) of tree species. Prominance values were calculated for shrubs and ground flora and were used for classification of the shrub and ground vegetation type. Higher area of the reserve was covered by the forest $(85.9 \%)$ followed by agriculture and buildup land $(5.1 \%)$, grassland (3\%), water body (3\%), riverbed (2\%) and shrub land (1\%), respectively. Based on the phyto-sociological combination, forests of this reserve incorporated four types namely Sal with Tarai Mixed Hardwood, Sal, Tarai Mixed Hardwood and Riverine.Ample of evidence found inside lowler part (Tarai, Bhabar and the base of Churia) of Parsa Wildlife Reserve. A suitable habitat with palatable forages (eg, Mallotus philippinnesis, Bananas, bamboos and climbers) for wild elephants were found inside the reserve. These forests were used as prime habitat by the Asiatic wild elephant.
\end{abstract}

Key words: Parsa wildlife reserve, wild asiatic elephant, importance value, prominance value, forest types.

\section{INTRODUCTION}

Aiming to preserve the population of indigineous Asiatic wild elephant (Elephus maximus) with associated flora and fauna of Parsa Wildlife Reserve of Nepal was gagetted in 1984. Geographically the reserve is located within $27^{\circ} 15^{\prime}-27^{\circ} 33^{\prime} \mathrm{N}$ latitude and $84^{\circ} 41^{\prime}-84^{\circ} 58^{\prime} \mathrm{E}$ longitude by occupying $499 \mathrm{~km}^{2}$ core and 298.17 $\mathrm{km}^{2}$ buffer zone areas (DNPWC 2006). Major portions of the reserve occupy Churia and Bhabar zone and also it supports the conservation of spill over wildlife of the Chitwan National Park in the east. The reserve includes tropical and sub-tropical forests of Parsa, Makwanpur and Bara districts in central lowland and churia in Nepal. The reserve lies in the humid sub-tropical climatic zone and exhibits four distinct seasons summer, monsoon, winter and spring. The summer (April-June) is 
intensely hot $\left(40^{\circ} \mathrm{C}\right)$ and humid with scarcity of water. The rainy season (July-September) is dominated by monsoon clouds and rains with little decline in the temperature. Characterized by very cold evenings and mornings $\left(7.7^{0} \mathrm{C}\right)$ with clear skies, winter lasts from October to December. Spring (January-March) is the enjoyable season with chilly nights and pleasing day temperatures and clearer skies (Chaudhary 1995).

Asiatic wild elephant (Elephus maximus), the largest megaherbivores in Asia is listed as protected species by the National Parks and Wildlife Conservation Act of Nepal (1973) (Chalise 2008). It has listed as a threatened speices in CITES appendix I. This species has been also listed in the World Conservation Unions (IUCN) list of threatened species since 1986 (IUCN 2006). Loss and fragmentation of habitats, poaching, domestication, and conflicts with human decline the wild elephants significantly over the last century (Yadav and Chalise 2013a,b, Sukumar 1989, Blake and Hedges 2004). Megaherbivores are mostly compreesed in the protected areas which are too small for long term population preservance (Owen-Smith 1988, Sukumar 1989). Currently 34500 to 51000 Asiatic wild elephants live in wild through out the range countries (Hedges et al. 2005).

There are four fragmented wild Asian elephants population in lowland harbor large and continuous, resident population of elephants (Yadav 2002, Yadav 2005a,b, Smith and Mishra 1992). Nonetheless, Malaria eradication program in 1950s resulted in heavy loss of continuous forestland for settlement purposes (Shrestha 1979), which restricted the elephants in four small partially or completely isolated fragments less than 200 animals each.

Wildlife like Elephants and other mega herbivores need more forage for foods and space than other small harbivores (Owen-Smith 1988). For such species, where population regulation through natural redution is not common forage is the likely limiting resource (Sinclair 1975, OwenSmith 1988, Sukumar 1989). Preference of food and habitat varies with season (Owen-Smith 1988, Sukumar 1989, Blom et al. 2004). The quantity and quality of food varies with the forest types, as determined by general productivity factors. Within the same forest type, local site factors determined the distribution of food plants (Pradhan 2007). African elephants tend to concentrate around permanent water sources during the dry season (Luethold 1977, Barnes and Hamilton 1982, Owen-Smith 1988), and they annually avoid human disturbance. Increasing numbers of elephants in a small area will alter the abundance and compositon of tree species (Law 1970, Croze 1974, Field 1976, Ben-Shahar 1998, Wiseman et al. 2004). Wild elephants mostly affect trees for feeding, and types of impact determine the intensity of damage to the forest. Trees grow slowly with secondary chemical defence in poor nutrient site (Fine et al. 2004, Marquis 2004). So the elephants are likely to feed more selectively in forest on nutrient poor sites than in richer forests. Jachmann and Bell (1985) inferred that the elephants improve their own habitat by knocking down trees in less preferred areas which promote secondary growth from coppicing. Elephants normally prefer secondary forest because of the ample amount of the food available in younger forest (Eltringham 1982).

The diverse representation of habitats of this reserve also provided shelters for different flora and fauna including endangered species like wild elephant (Elephus maximus), tiger (Panthera tigris) and occassional visiting rhino (Rhinoceros unicornis). The reserve and surrounding forests is sole area that conserve and manage the indigineous resident population of Asiatic wild elephant in Nepal. This study provided the quantitative analysis of the available habitats in the Parsa Wildlife Reserve and its utilization by the Asiatic wild elephant (DNPWC 2006). 


\section{METHODS}

Grids of $2 \times 2 \mathrm{~km}(\mathrm{n}=181)$ were prepared for the whole reserve and buffer zone area. Plots were systematically distributed inside each cluster. From the designed grid, $5.5 \%$ were selected randomly to conduct inventory. One grid incorporated a cluster of 10 quadrats arranged diagonally from southwest to north-east corner of the grid. The plots are located between $150 \mathrm{~m}$ to each other. Circular plots were used to conduct forest inventory (Yadav 1988, 2005). Plots with $10 \mathrm{~m}$ radius were used to collect information on tree level characteristics. Plots with $2 \mathrm{~m}$ radius were used to collect information about shrubs and plots with $1 \mathrm{~m}$ radius were used to collect information about grass and other herbaceous plants. Characteristics like local name, scientific name, DBH and height were recorded for trees. For the shrubs information like local name, scientific name and crown cover for each species were collected. Information like local name, scientific name and coverage of each species were collected for herbaceous species and grass.

Frequency of each species was analyzed by dividing the number of quadrats in which the specific species occurred by total number of quadrats. Similarly, the percentage ratio of frequency of particular species with the sum of frequencies of all species was relative frequency. Density, the number of individuals per unit area, was calculated by dividing number of individuals of particular species by total area (ha) of the studied quadrats. Relative density was the percentage ratio of density of one species with sum of densities of all species. Dominance, amount of ground covered by the tree trunk, was the ratio of total basal area of particular species with the total area (ha) of the studied quadrats. The relative amount of ground covered by the tree trunk called as relative dominance was the percentage ratio of the dominance of particular species with the total dominance of all species. Importance values of each species of trees were analyzed by adding relative frequency, relative density and relative dominance. Name of each forest type was determined from the sequencial order of the large Importance Values (IV) of tree species (Zobel et al. 1987). Prominance value (PV) was calculated for shrub and ground flora (like grass and herbaceous species). Prominance values were the multiplication of mean percent covers of particular species with the square roots of its frequency. These values were used for classification of the shrub and ground vegetation type from the sequencial order of large PV (Sharma et al. 2012).

Forest types were determined on the basis of density of the tree in canopy layer and occurrence locality of the forest (HMGN 1999). On those bases the forest types were recognized as follows:

Sal with Tarai Mixed Hardwood forest: In this forest, different tree species are present along with the Sal (Shorea robusta). In the forest the percentage relative basal area of Sal was $30-60 \%$.

Sal forest: In this forest Sal was the dominant species. In this forest more than $60 \%$ relative basal area was represented by Sal (Shorea robusta).

Tarai Mixed Hardwood forest: This forest included different tree species. If the basal area representation of Sal is less than $30 \%$ it was also considered as Tarai mixed hardwood forest.

Riverine forest: This forest was available in the moist area mostly in the river side.

Presence of wild elephants was recorded through identifying traditional route (Hatti dandi) of elephants that is used as transect. Recording of foot prints, dungs and direct observations of wild elephants were carried out along to transect $50 \mathrm{~m}$ apart both side. Damage of trees by elephants was also recorded. Vegetation analyses were carried out in PWR with respect to elephants use. Species grazed and browsed by wild elephants were recorded during vegetation survey in PWR. 


\section{RESULTS}

Total numbers of surveyed plots were 297. Among them 99 plots were used to collect tree characteristics, 99 plots were used to collect information on shrubs and 99 plots were used to collect data about herbaceous plant and grasses. The altitude of the surveyed plots varied from 121 $\mathrm{m}$ to $782 \mathrm{~m}$.

Along the designed transects, the study team observed, and recorded ample of foot prints and dungs of wild elephants in lowland designated grids. The team did not observe or recorded sinage of elephant's foot prints and dungs in Churia hills. Sinage of elephants only recorded in Bhabar zone and lower part of Churia hills. More than 30 different forage species grazed, browsed and knocked by elephants were listed during the study. The tree species were Ficus lacor, Acacia hispide, Musa sapientum, Ficus benjamina, Garuga pinnata, Dandrocalamus spp., Artocarpus lakoocha, Acacia catechu, Bombax cieba, Dalbergia sissoo, Dillenia pentagyna, Ficus benghalensis, Ficus racemosa, Ficus religiosa, Litsea monopetala, Mallotus philippinensis, Shorea robusta, Terminalia belerica, Terminalia chebula, Calamus tenuis, Circium wallichi.

Grass species were Arundodonax, Desmostachya bipinnata, Imperata cylindrica, Phragmites kharka, Saccharum bengalensis, Saccharum spontaneum, Typha elephentina, Vetiveria zizanoides and the very few Spatholobus parviflorus and Bauhinia vellai (Annex 2).

On the basis of the plot location the land use types of Parsa Wildlife Reserve and its buffer zone were analyzed. Higher land area of the reserve is occupied by the forest $(85.9 \%)$ followed by agriculture and buildup land (5.1\%), grassland $(3 \%)$, water body $(3 \%)$, riverbed $(2 \%)$ and shrub land $(1 \%)$, respectively (Table 1$)$.

The aquatic habitat incorporated about $5 \%$ of the study area. The East -- Rapti river is the one of the main aquatic habitat of PWR in the north of the reserve. Bhatta Khola and Sital Khola are other aquatic habitats of this reserve. Likewise Laukidah, Dewaki Daha are also the aquatic habitat of this reserve.

Table 1. Land use type in the Parsa Wildlife Reserve and its buffer zone.

\begin{tabular}{lc}
\hline Land use category & Percentage \\
\hline Forest & 85.9 \\
Agriculture and buildup land & 5.1 \\
Grassland & 3.0 \\
Water & 3.0 \\
Riverbed & 2.0 \\
Shrubland & 1.0 \\
Total & 100.0 \\
\hline
\end{tabular}

Terrestrial habitat of the reserve incorporated three vegetation types namely forest, shrubland and grassland. Based on the phyto-sociological combination, forests of this reserve are divided in four categories. They are Sal withTarai Mixed Hardwood, Sal, Tarai Mixed Hardwood and Riverine forests.

The largest area of the reserve is occupied by Sal withTarai Mixed Hardwood (34.8\%) followed by Tarai Mixed Hardwood forest $(30.3 \%)$, Sal forest $(24.7 \%)$ and Riverine forest (5.6\%), respectively (Table 2). Small patches of Sal-salla (Shorea robusta-Pinus roxburghii) forest and Salla forest (Pinus roxburghii) also exist in some higher altitude of the reserve.

Table 2. Vegetation types in Parsa Wildlife Reserve and its buffer zone.

\begin{tabular}{lc}
\hline Vegetation & Occupied area $(\boldsymbol{\%})$ \\
\hline $\begin{array}{l}\text { Sal with Tarai Mixed } \\
\quad \text { Hardwood forest }\end{array}$ & 34.8 \\
Tarai Mixed & \\
$\quad$ Hardwood forest & 30.3 \\
Sal forest & 24.7 \\
Riverine forest & 5.6 \\
Grass land & 3.4 \\
Shrub land & 1.1 \\
Total & 100.0 \\
\hline
\end{tabular}


In the canopy layer of Sal with Tarai mixed hardwood (STMH) forest, 36 species of tree were identified from this forest. In this forest importance value of Sal (Shorea robusta) is highest (110.8) followed by Lagerstroemia parviflora (37.5) and Dillenia pentagyna (16.2), respectively. The understory layer provided good hiding areas for the wildlife. In this study shrubs were considered as understory layer. Total number of shrub species recorded for understory was 23. Among them Desmodium heterocarpon was more prominent ( $\mathrm{PV}=6.23$ ) followed by Woodfordia fruticosa $(\mathrm{PV}$ $=4.40)$. Ground vegetation is the important food for the wild elephant and wild herbivores. This vegetation has an important role in maintaining the food chain of the reserve for wildlife. In total 34 species of plants were recorded as ground flora of STMH forest. On the basis of prominence value, more prominent ground flora of this forest were Imperata cylindrica ( $\mathrm{PV}=58.2)$ followed by Saccharum spontaneum (33.9) and Arundinella nepalensis (10.9), respectively (Table 3).

From the canopy layer of Sal forest 21 species of tree were reported. In this forest importance value of Sal (Shorea robusta) is highest (189.0) followed by Lagerstroemia parviflora (16.0) and Anogeissus latifolia (11.4), respectively. In the understory layer total number of species recorded for shrub was 21. Among the recorded shrub species, Phoenix humilis was more prominent (PV $=16.8$ ) followed by Woodfordia fruticosa $(\mathrm{PV}=$ 7.9) and Desmodium heterocarpon ( $\mathrm{PV}=3.8)$, respectively. Ground vegetation of this forest incorporated 23 species of plants. On the basis of prominence value, more prominent ground flora of this forest was Cyperus compressus $(\mathrm{PV}=28.5)$ followed by Themeda arundinacea $(\mathrm{PV}=21.3)$ and Eulaliopsis binata $(\mathrm{PV}=15.6)$, respectively (Table 3).

The canopy tree of Tarai mixed hardwood (TMH) forest included 18 species. In this forest importance value of Shorea robusta is highest
(37.0) followed by Terminalia alata (25.4) and Lagerstroemia parviflora (22.3), respectively. This forest incorporated 22 species of shrubs. Among the recorded shrub species Woodfordia fruticosa was more prominent $(\mathrm{PV}=6.7)$ species followed by Caesalpinia decapetala $(\mathrm{PV}=2.4)$ and Colebrookea oppositifolia $(\mathrm{PV}=1.9)$, respectively. In total 23 species of plants were recorded as ground flora of TMH forest. On the basis of prominence value, more prominent ground flora of this forest was Eupatorium odoratum $(\mathrm{PV}=63.6)$ followed by Imperata cylindrica (31.6) and Themeda arundinacea (24.6), respectively (Table 3).

From the canopy layer of riverine forest 13 species of tree were identified. In this forest importance value of Adina cordifolia was highest (60) followed by Garuga pinnata (41.9) and Bombax ceiba (36.6), respectively. Total number of species recorded for shrub was 4 . In the shrub layer Caesalpinia decapetala was more prominent species $(\mathrm{PV}=2.2)$ followed by Pogostemon benghalensis $(\mathrm{PV}=0.4)$, Ardisia solanacea $(\mathrm{PV}=$ 0.3 ) and Letsea macrophylla (PV = 0.1), respectively. In total 10 species of plants were recorded as ground flora of riverine forest. On the basis of prominance value, more prominent ground flora of this forest was Saccharum spontaneum $(\mathrm{PV}=189)$ followed by Themeda arundinacea (49.2) and Heteropogon contortus (31.3), respectively (Table 3 ).

From the grassland of Parsa Wildlife Reserve three species of shrubs were recorded. The Colebrookea oppositifolia was more prominent shrub (PV = 4.8) species followed by Pogostemon benghalensis $(\mathrm{PV}=0.8)$ and Melastoma melabathricum $(\mathrm{PV}=0.6)$. In total 10 species of plants were recorded as ground flora of grassland. On the basis of prominence value, more prominent ground flora of this vegetation was Cynodon dactylon $(\mathrm{PV}=365.3)$ followed by Ageratum conyzoides (270.8) and Digitaria ciliaris (108.9), 
respectively. From the shrubs land of PWR five of plants were recorded as ground flora of shrub species of shrub were recorded.In total 10 species land (Table 3).

Table 3. Species recorded from Parsa Wildlife Reserve with their Importance Value (IV) for tree and Prominance Value (PV) for shrub and herbaceous species.

\begin{tabular}{|c|c|c|c|c|c|c|}
\hline SN & Scientific name & Local name & STMH & TMH & Sal & Grassland \\
\hline 1 & Achyranthes aspera & Datiwan & 6.2 & 1.3 & 0.0 & 0.0 \\
\hline 2 & Adina cordifolia & Kadam & 58.2 & 31.6 & 0.0 & 0.0 \\
\hline 3 & Ageratum conyzoides & Gandejhar & 1.0 & 13.4 & 270.8 & 0.0 \\
\hline 4 & Ajuga bracteosa & Ratpate & 0.2 & 0.0 & 0.2 & 0.0 \\
\hline 5 & Albizia procera & Setosiris & 0.8 & 0.8 & 0.0 & 0.0 \\
\hline 6 & Anogeissus latifolius & Dhauti & 0.0 & 0.0 & 11.4 & 0.0 \\
\hline 7 & Ardisia solanacea & Bhanti & 0.0 & 0.6 & 0.7 & 0.0 \\
\hline 8 & Artemisia indica & Titepati & 4.4 & 6.7 & 0.0 & 38.5 \\
\hline 9 & Arundinella nepalensis & Kharuki, Musekhari & 0.1 & 0.1 & 11.2 & 0.0 \\
\hline 10 & Asparagus filicinua & Kurilo & 0.0 & 0.0 & 1.5 & 0.0 \\
\hline 11 & Barleria cristata & Bhendekuro & 4.7 & 16.4 & 0.0 & 0.0 \\
\hline 12 & Bauhinia purpurea & Tanki & 2.1 & 1.6 & 0.0 & 0.0 \\
\hline 13 & Bauhinia vahlii & Bhorla & 0.2 & 0.3 & 0.0 & 0.0 \\
\hline 14 & Bauhinia variegate & Koiralo & 0.0 & 0.0 & 3.1 & 0.0 \\
\hline 15 & Bombax ceiba & Simal & 1.8 & 6.3 & 0.0 & 0.0 \\
\hline 16 & Buchanania latifolia & Piyari & 0.0 & 0.0 & 4.9 & 0.0 \\
\hline 17 & Caesalpinia decapetala & Arelikanda & 1.1 & 0.5 & 0.5 & 0.0 \\
\hline 18 & Calopogonium macunoides & Gahatejhar & 2.1 & 3.1 & 0.0 & 0.0 \\
\hline 19 & Carex cruciata & Harkatta (Khar) & 1.0 & 3.3 & 0.0 & 0.0 \\
\hline 20 & Careya arborea & Kumbhi & 1.0 & 1.1 & 3.0 & 0.0 \\
\hline 21 & Casearia elliptica & Ber & 0.7 & 0.0 & 0.0 & 0.0 \\
\hline 22 & Casearia graveolens & Badkaule, Piperi & 0.0 & 0.0 & 5.5 & 0.0 \\
\hline 23 & Cassia fistula & Rajbriksha & 0.1 & 0.0 & 0.0 & 0.0 \\
\hline 24 & Cassia tora & Chinchin & 3.8 & 7.1 & 0.0 & 0.0 \\
\hline 25 & Cautleya spicata & Panisaro & 10.0 & 1.5 & 0.0 & 0.0 \\
\hline 26 & Cheilanthes dalhousiae & Rani sinka & 0.0 & 0.0 & 2.1 & 0.0 \\
\hline 27 & Chlorophytum arundinaceum & Dati sag & 0.0 & 0.0 & 0.0 & 0.0 \\
\hline 28 & Cissampelos pareira & Batul pate & 0.0 & 0.0 & 0.0 & 0.0 \\
\hline 29 & Cissus repens & Gujargano, Pureni & 3.3 & 0.0 & 0.0 & 0.0 \\
\hline 30 & Clausena pentaphylla & Raunne & 2.2 & 9.1 & 0.0 & 0.0 \\
\hline 31 & Cleistocalyx operculatus & Kyamuna, Phandir & 3.2 & 0.4 & 2.5 & 0.0 \\
\hline 32 & Cleome viscosa & Bantori & 0.0 & 0.0 & 0.5 & 0.0 \\
\hline 33 & Colebrookea oppositifolia & Dhursul & 0.0 & 0.0 & 0.0 & 4.8 \\
\hline 34 & Commelina benghalensis & Kanejhar & 5.5 & 10.5 & 0.2 & 0.0 \\
\hline 35 & Corchorus capsularis & Patuwa & 11.9 & 4.9 & 0.0 & 0.0 \\
\hline 36 & Cornus oblonga & Latikath & 0.1 & 0.1 & 2.4 & 0.0 \\
\hline 37 & Crinum amoenum & Ban pyaj & 0.5 & 2.4 & 0.0 & 0.0 \\
\hline 38 & Curculigo orchioides & Musali & 0.1 & 1.3 & 1.8 & 0.0 \\
\hline 39 & Cynodon dactylon & Dubo & 0.1 & 0.0 & 0.0 & 365.3 \\
\hline 40 & Cyperus compressus & Mothe & 0.0 & 0.0 & 28.5 & 43.0 \\
\hline 41 & Dalbergia latifolia & Satisal & 9.9 & 24.6 & 0.0 & 0.0 \\
\hline 42 & Dennstaedtia appendiculata & Uniu & 0.0 & 0.0 & 12.4 & 0.0 \\
\hline 43 & Desmodium elegans & Bakhreghans & 0.0 & 4.0 & 0.0 & 0.0 \\
\hline 44 & Desmodium heterocarpon & Sakhino & 0.0 & 6.4 & 3.9 & 0.0 \\
\hline 45 & Desmodium oojenense & Sandan & 0.0 & 0.0 & 5.3 & 0.0 \\
\hline 46 & Desmodium sp. & Desmodiumsp & 0.0 & 1.6 & 0.0 & 0.0 \\
\hline
\end{tabular}




\begin{tabular}{|c|c|}
\hline 47 & Desmostachya bipinnata \\
\hline 48 & Digitaria ciliaris \\
\hline 49 & Dillenia pentagyna \\
\hline 50 & Dioscorea pentaphylla \\
\hline 8 & Ehretia laevis \\
\hline 52 & Elaeagnus infundibularis \\
\hline 53 & Elatostema platyphyllum \\
\hline 54 & Elephantopus scaber \\
\hline 55 & Elsholtzia blanda \\
\hline 56 & Equisetum diffusum \\
\hline 57 & Eulaliopsis binata \\
\hline 58 & Eupatorium adenophorum \\
\hline 59 & Eupatorium odoratum \\
\hline 60 & Eurya acuminate \\
\hline 61 & Ficus hispida \\
\hline 62 & Flemingia macrophylla \\
\hline 63 & Flemingia strobilifera \\
\hline 64 & Garcinia xanthochymus \\
\hline 65 & Garuga pinnata \\
\hline 66 & Gmelina arborea \\
\hline 67 & Gouania leptostachya \\
\hline 68 & Grewia subinaequalis \\
\hline 69 & Heteropogon contortus \\
\hline 70 & Hymenodictyon excelsum \\
\hline 71 & Imperata cylindrica \\
\hline 72 & Inula cappa \\
\hline 73 & Justicia adhatoda \\
\hline 74 & Lagerstroemia parviflora \\
\hline 75 & Lannea coromandelica \\
\hline 76 & Leea macrophylla \\
\hline 77 & Leucas sp. \\
\hline 78 & Litsea monopetala \\
\hline 79 & Luculia gratissima \\
\hline 80 & Lygodium flexuosum \\
\hline 81 & Madhuca latifolia \\
\hline 82 & Maesa chisia \\
\hline 83 & Melastoma melabathricum \\
\hline 84 & Melia azedarach \\
\hline 85 & Millettia extensa \\
\hline 86 & Mucuna nigricans \\
\hline 87 & Murraya koenigii \\
\hline 88 & Nephrolepis auriculata \\
\hline 89 & Nyctanthes arbor-tristis \\
\hline 90 & Parthenocissu ssemicordata \\
\hline 91 & Phoenix humilis \\
\hline 92 & Phyllanthus emblica \\
\hline 93 & Phyllanthus parvifolius \\
\hline 94 & Pilea sp. \\
\hline 95 & Pinus roxburghii \\
\hline 96 & Pinus sp \\
\hline 97 & Pogostemon benghalensis \\
\hline 98 & Premna barbata \\
\hline
\end{tabular}

Kush
Chitrebanso
Tantari
Ban tarul
Datrung
Gunyalo
Gagleto
Marcha
Ban silam
Ankhle
Babio
Banmara
Banmara
Jhigane
Khasreto
Bhatte
Flemengiasp
Archal
Dabdabe
Khamari
Bhatmase
Phorso
Arthunge
Bhudkul
Siru
Gaitihare
Asuro
Botdhainyaro
Hallunde, Jhigat
Galeni
Leucassp
Kutmero
Kangiyo
Janailahara
Latimauwa
Bilaune
Kali angeri
Bakaino
Gaujo
Kauso
Karipatta
Paniamala
Parijat
Charchare
Thakal
Amala
Khareto
Kamle
Khotesalla
Sallo
Rudilo
Gindari

\begin{tabular}{|c|c|c|c|}
\hline 0.0 & 0.0 & 1.9 & 43.0 \\
\hline 0.0 & 0.0 & 0.1 & 108.9 \\
\hline 0.0 & 0.0 & 9.8 & 0.0 \\
\hline 1.0 & 0.0 & 0.0 & 0.0 \\
\hline 10.3 & 16.1 & 0.0 & 0.0 \\
\hline 0.0 & 0.0 & 0.0 & 0.0 \\
\hline 0.0 & 0.0 & 0.0 & 0.0 \\
\hline 0.1 & 0.1 & 3.7 & 0.0 \\
\hline 0.0 & 0.0 & 0.0 & 0.0 \\
\hline 6.2 & 13.5 & 0.2 & 47.1 \\
\hline 3.5 & 0.0 & 15.6 & 0.0 \\
\hline 1.0 & 0.0 & 0.4 & 27.2 \\
\hline 2.1 & 6.8 & 8.5 & 0.0 \\
\hline 3.1 & 9.6 & 0.0 & 0.0 \\
\hline 0.0 & 0.0 & 0.2 & 0.0 \\
\hline 0.0 & 0.0 & 0.2 & 0.0 \\
\hline 0.7 & 0.6 & 0.0 & 0.0 \\
\hline 1.4 & 0.0 & 0.0 & 0.0 \\
\hline 9.3 & 16.4 & 0.0 & 0.0 \\
\hline 0.0 & 3.3 & 0.0 & 0.0 \\
\hline 0.0 & 0.0 & 0.1 & 0.0 \\
\hline 110.8 & 37.0 & 2.4 & 0.0 \\
\hline 11.3 & 0.9 & 10.0 & 27.2 \\
\hline 0.1 & 0.0 & 0.0 & 0.0 \\
\hline 0.0 & 0.0 & 5.9 & 43.0 \\
\hline 3.6 & 0.0 & 0.3 & 0.0 \\
\hline 0.7 & 4.9 & 0.2 & 0.0 \\
\hline 0.1 & 0.0 & 16.0 & 0.0 \\
\hline 2.7 & 13.4 & 0.0 & 0.0 \\
\hline 2.7 & 63.6 & 0.3 & 0.0 \\
\hline 0.2 & 0.0 & 0.0 & 0.0 \\
\hline 0.0 & 0.0 & 0.0 & 0.0 \\
\hline 2.0 & 0.1 & 0.0 & 0.0 \\
\hline 7.4 & 3.2 & 0.0 & 0.0 \\
\hline 4.1 & 5.0 & 0.0 & 0.0 \\
\hline 0.0 & 0.0 & 0.1 & 0.0 \\
\hline 0.1 & 0.0 & 0.1 & 0.6 \\
\hline 2.7 & 1.4 & 0.0 & 0.0 \\
\hline 0.0 & 0.0 & 0.6 & 0.0 \\
\hline 2.0 & 0.0 & 0.0 & 0.0 \\
\hline 0.0 & 0.0 & 1.0 & 0.0 \\
\hline 33.9 & 19.0 & 0.0 & 0.0 \\
\hline 0.0 & 3.6 & 0.0 & 0.0 \\
\hline 0.1 & 0.0 & 0.0 & 0.0 \\
\hline 6.9 & 0.0 & 16.8 & 0.0 \\
\hline 5.4 & 10.9 & 2.3 & 0.0 \\
\hline 0.0 & 0.3 & 0.0 & 0.0 \\
\hline 37.5 & 22.3 & 0.0 & 0.0 \\
\hline 0.0 & 1.5 & 0.0 & 0.0 \\
\hline 6.2 & 1.6 & 7.9 & 0.0 \\
\hline 9.8 & 25.4 & 0.1 & 0.8 \\
\hline 0.0 & 0.1 & 0.0 & 0.0 \\
\hline
\end{tabular}




\begin{tabular}{|c|c|c|c|c|c|c|}
\hline 99 & Psidium guajava & Amba & 2.2 & 8.3 & 0.0 & 0.0 \\
\hline 100 & Pueraria tuberose & Biralilaharo & 0.0 & 1.9 & 0.0 & 0.0 \\
\hline 101 & Rabdosia lophanthoides & Charpate & 1.6 & 0.0 & 0.0 & 0.0 \\
\hline 102 & Rubus ellipticus & Ainselu & 0.3 & 0.6 & 0.0 & 0.0 \\
\hline 103 & Rubus niveus & Ratoainselu & 2.2 & 3.2 & 0.0 & 0.0 \\
\hline 104 & Saccharum spontaneum & Kans & 0.1 & 0.0 & 3.9 & 86.0 \\
\hline 105 & Schefflera venulosa & Kursimal & 0.3 & 0.0 & 0.0 & 0.0 \\
\hline 106 & Schima wallichii & Chilaune & 0.0 & 0.0 & 2.3 & 0.0 \\
\hline 107 & Schleichera oleosa & Kusum & 2.8 & 2.1 & 0.0 & 0.0 \\
\hline 108 & Semecarpus anacardium & Bhalayo & 0.6 & 2.2 & 8.8 & 0.0 \\
\hline 109 & Senecio cappa & Marchajhar & 0.0 & 0.0 & 0.0 & 33.3 \\
\hline 110 & Shorea robusta & Sal & 0.0 & 7.8 & 189.0 & 0.0 \\
\hline 111 & Smilax aspera & Kukurdaino & 6.4 & 9.3 & 0.0 & 0.0 \\
\hline 112 & Sonchus asper & Sonchusaspera & 0.1 & 0.1 & 0.2 & 0.0 \\
\hline 113 & Spatholobus parviflorus & Debrelahara & 0.2 & 0.1 & 0.0 & 0.0 \\
\hline 114 & Sterculia villosa & Odal & 0.0 & 0.0 & 0.0 & 0.0 \\
\hline 115 & Swertia angustifolia & Chiraito & 1.0 & 4.9 & 0.0 & 0.0 \\
\hline 116 & Syzygium cumini & Jamun & 7.4 & 9.3 & 7.0 & 0.0 \\
\hline 117 & Terminalia alata & Asna & 2.4 & 4.5 & 7.7 & 0.0 \\
\hline 118 & Terminalia bellirica & Barro & 1.0 & 0.0 & 2.3 & 0.0 \\
\hline 119 & Terminalia chebula & Harro & 0.0 & 0.0 & 2.4 & 0.0 \\
\hline 120 & Themeda arundinacea & Dhaddi & 4.8 & 9.3 & 21.3 & 0.0 \\
\hline 121 & Thespesia lampas & Ban kapas & 3.9 & 3.4 & 0.0 & 0.0 \\
\hline 122 & Thysanolaena maxima & Amliso & 0.7 & 3.6 & 0.0 & 0.0 \\
\hline 123 & Toona ciliatae & Tooni & 0.0 & 1.4 & 0.0 & 0.0 \\
\hline 124 & Trichilia connaroides & Ankhataruwa & 2.1 & 0.0 & 0.0 & 0.0 \\
\hline 125 & Unknown & Chabo & 0.6 & 0.2 & 0.0 & 0.0 \\
\hline 126 & Unknown & Kharkhucho & 6.8 & 11.2 & 0.0 & 0.0 \\
\hline 127 & Unknown & 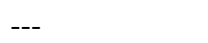 & 0.0 & 1.4 & 3.9 & 0.0 \\
\hline 128 & Vitex negundo & Simali & 0.0 & 0.0 & 0.0 & 0.0 \\
\hline 129 & Wightia speciosissima & Phurkeghaans & 0.0 & 0.0 & 0.3 & 0.0 \\
\hline 130 & Woodfordia fruticosa & Dhairo & 16.2 & 13.0 & 8.0 & 0.0 \\
\hline 131 & Zizyphus incurve & Hade bayar & 0.0 & 0.1 & 0.0 & 0.0 \\
\hline 132 & Zizyphus mauritiana & Bayar & 1.1 & 0.0 & 0.0 & 0.0 \\
\hline 133 & Zizyphus rugosa & Jungalibayar & 2.1 & 0.0 & 0.0 & 0.0 \\
\hline
\end{tabular}

Note: STMH - Sal mixed Tarai Mixed Hardwood, TMH - Tarai Mixed Hardwood

Table 4. Common species known to be eaten by elephants (grazed, browsed or eaten).

\begin{tabular}{llll}
\hline Scientific name & Nepali name & Plant Ecology & Most common vegetation type (s) \\
Tree species & & & \\
Ficus lacor & Kabhro & Tree & Mixed forest \\
Mussa sapientum & Babul & Tree & Mixed forest \\
Ficus benjamina & Banana & Tree & Mixed forest (Riverine) \\
Ficus semicordata & Swami & Clump & Mixed forest (Riverine) \\
Garuga pinnata & Khnyo & Tree & Mixed forest (Riverine) \\
Dendrocalamus sp & Dabdabe & Tree & Mixed forest (Riverine) \\
Artocarpus lakoocha & Bans & Clump & Riverine forest
\end{tabular}




\begin{tabular}{|c|c|c|c|}
\hline Acacia catechu & Khair & Tree & Khair-Sisoo forest \\
\hline Bombax cieba & Simal & Tree & Mixed hardwood forest, wooded grassland \\
\hline Dalbergia sissoo & Sisoo & Tree & Khair-Sisoo forest \\
\hline Dillenia pentagyna & Tantari & Tree & Sal (Shorea robusta) forest \\
\hline Dryothyrium boryanum & Kochina (Neoro) & Tree & --- \\
\hline Ficus affinis & Gajahar & Tree & Sal (Shorea robusta) forest \\
\hline Ficus benghalensis & Bara & Tree & Sal (Shorea robusta) forest \\
\hline Ficus glumerata & Gular & Tree & Riverine forest \\
\hline Ficus religiosa & Pipal & Tree & --- \\
\hline Litsea monopetala & Kutmiro & Tree & --- \\
\hline Mallotus philippinensis & Sindure & Tree & Riverine forest \\
\hline Shorea robusta & Sal & Tree & Sal (Shorea robusta) forest \\
\hline Terminalia belerica & Harro & Tree & Sal (Shorea robusta) forest \\
\hline Terminalia chebula & Barro & Tree & Sal (Shorea robusta) forest \\
\hline Calamus tenuis & Bent & Shrub & Riverine forest \\
\hline Circium wallichi & GaindaKhar & Herb & --- \\
\hline \multicolumn{4}{|l|}{ Grass species } \\
\hline Arundo donax & ThuloNarkat & Grass & Grassland \\
\hline Desmostachya bippinata & Kush & Grass & Grassland \\
\hline Imperata cylindrica & Siru & Grass & Grassland \\
\hline Phragmites karka & Narkat & Grass & Grassland \\
\hline Saccharum bengalensis & Baruwa & Grass & Grassland \\
\hline Saccharum spontaneum & Senth (Kansh) & Grass & Grassland \\
\hline Typha elephentina & Pater & Grass & Grassland \\
\hline Vetiveria zizannoides & Kansh & Grass & Grassland \\
\hline \multicolumn{4}{|l|}{ Climbers (vines) } \\
\hline Spatholus roxburghii & Debre Lahara & Climber & Sal (Shorea robusta) forest \\
\hline Bauhunia valhlii & Bhorla & Climber & Sal (Shorea robusta) forest \\
\hline
\end{tabular}

\section{DISCUSSION}

In the previous study of the core zone of PWR (DNPWC 2006) the land use incorporated forest lands $(91.78 \%)$, cultivated land $(0.43 \%)$, shrub land $(0.45 \%)$, and river or sand $(7 \%)$. This study also incorporated the buffer zone areas so the land use has been changed. The area of forest land was reduced nearly by $5 \%$ and simultaneously increased in agriculture and builtup land nearly by $4.5 \%$. In the previous study there was no incorporation of the grassland, but current study revealed that the reserve incorporated $1 \%$ area as grassland. The cultivated land inside the reserve
(Rambhori and Bhatta) were shifted to the outside location of PWR.Two villages (Ramauli and Pratappur) of Manahari VDCs of Makwanpur have evacuated from PWR core areas in 2013. About 200 ha of cultivated land and about 10 ha of settlement land has been converted into grassland. Currently those cultivated land was converted into grassland. So, there is increase in the grassland area inside the reserve. This study also reveled that there are slight increase in shrubland area. That is due to the incorporation of buffer zone in this study. 
In the previous study, Tarai mixed hardwood forest was named as mixed deciduous hardwood forest (Chaudhary 1995, BPP 1996). At the canopy layer tree composition of the TMHF resembles with the current findings. The species composition in the ground flora was differing from the previous record. The envasive species Eupatorium odoratum was found prominent in the ground flora. This indicated that the envasive flora are gradually arising in the study area.

The current riverine forest type was previously recognized as mixed deciduous riverine forest (Chaudhary 1995, BPP 1996). The species composition in the forest was changed from the previous record. The previously reported tree species Acacia catechu and Celtis tetranda were not recorded from this forest.

Tree species in the canopy layer of Sal forest was similar with the previous finding of Chaudhary (1995). The shrub species of the sal forest was not recorded in previous record. The ground vegetation of Sal forest was differing from the previous findings. Previously Curcuma leucorhiza and Costus speciosa were the main species but currently Cyperus compressus, Themeda arundinacea and Eulaliopsis binata were common as ground flora.

Previously mentioned forests like Sal-Pine (Shorea robusta-Pinus roxburghii), Pine (Pinus roxburghii) as well as Khair (Acacia catechu) (Chaudhary 1995, BPP 1996) were not reporded in this study. Instead of those forests the combine forest naming Sal with Tarai mixed hardwood forest was reported in this study.

Previously vegetations (Fodder trees, grasses and climbers) grazed, browsed and damage of trees by wild elephants was not identified. Plenty of forage for elephants have been found in Bhabar zone and lower part of the PWR during the vegetation survey.

\section{ACKNOWLEDGEMENT}

The authors acknowledge Department of National Parks and Wildlife Conservation and Parsa Wildlife Reserve for financial support with research permission. Thanks to Mr. Pashupati Adhikari for coordinating the field work activities and Mr. Promod Yadav for helping in data collection. Acknowledgement is expressed to Mr. Nilambar Mishra and Mr. Mohamad Manjur Ahamad for coordination and help. Thanks to Mr. Salikram Sigdel and Mr Ramesh Gautam for their hard work in data collection. Last but not least acknowledgement also goes to Mr. Bhola Dhakal GIS expert for his contribution in designing the Grids and sample plots by GIS method.

\section{REFERENCES}

Barnes, R.F.W. and I.D. Hamilton. 1982.The number and distribution patterns of large mammals in the Rauha-Rangwa area of southern Tanzania. Journal of Applied Ecology 19:411-425.

Ben-Shahar, R. 1998. Changes in structure of savanna woodlands in northern Botswana following the impacts of elephants and fire. Plant Ecology 136:189-194.

Blake, S. and S. Hedges. 2004. Sinking the flagship: The case of forest elephants in Asia and Africa. Conservation Biology 18:11911202.

Blom, A., R. van Zalinge, E. Mbea, I.M.A. Heitkoning and H.H.T. Prins. 2004. Human impact on wildlife populations within a protected Central African Forest. African Journal of Ecology 43:23-31.

BPP. 1996. An Assessment of the Representation of the Terrestrial Ecosystems within the Protected Area System of Nepal. Biodiversity

ECOPRINT VOL 20, 2013 
Profiles Project (BPP), Publication No. 15. Ministry of Forest and Soil conservation, HMGN, Katmandu, Nepal.

Chalise, M.K. 2008. Nepalka Samrakshit Banyajantu (Nepal's Protected Wildlife) (in Nepali). Shajha Prakashan, (A Corporate Publishing House), Lalitpur, Nepal, 116+12 pp.

Chaudhary, R.P. 1995. Biological and Cultural Diversity of Parsa Wildllife Reserve. Institute of Biodiversity, Kathmandu, Nepal.

Corze, H. 1974. The Seronrabull problem II, The trees. East Africa Wildlife Journal 12:29-47.

Eltringham, S.K. 1982. Elephants. Poole, Dorset, Blandford Press.

Field, C.R. 1976. The savana ecology of Kidepo Valley National Park, II, Feedingecology of elephant and giraffe. East African Wildlife Journal 14:1-15.

Fine, P.V.A., I. Mesones and P.D. Coley. 2004. Herbivores promote habitat specialization by trees in Amazonian forests. Science 305:663665.

Hedges, S., M.J. Tyson, A.F. Sitompul, D. Gunaryadi and Aslan. 2005. Distribution, status and conservation needs of Asian elephants (Elephus maximus) in Lampung province, Sumatra, Indonesia. Biological Conservation 124:35-48.

HMGN. 1999. Forest Resources of Nepal (19871998). Ministry of Forests and Soil Conservation, His Majesty's Government of Nepal, Kathmandu, Nepal.

IUCN. 2006. 2006 IUCN Red List of Threatened Species, WWW.iucnredlist.org.

Jachmann, H. and R.H.V. Bell. 1985. Utilization by elephants of the Brachystegia woodland of the Kasungu Natioal Park, Malawi. African Journal of Ecology 23:245-258.

Laws, R.M. 1970. Elephants as agents of habitat and landscape change in East-Africa. Oikos 21:1-15.

Luethold, W. 1977. Spatial organization and strategy of habitat utilization of elephant in Tsavo National Park Kenya. Zeitchrift Saugetierkunde 42:358-379.

Marquis, R.J. 2004. Herbivores rule. Science 305:619-621.

Owen-Smith, R.N. 1988. Megaherbivores: The Influence of Very Large Body Size in Ecology. Cambridge University Press, Cambridge, 369 pp.

Pradhan, N.M.B. 2007. An Ecological Study of a Re-colonizing Population of Asian Elephants (Elephas maximus) in Lowland Nepal.

Sharma, B.K., M.K. Chalise and G.S. Solanki. 2012. Vegetation types and wildlife occurrence in Baghmara Buffer Zone Community Forest, Nepal. International Multidisciplinary Research Journal 2(2):5265.

Shrestha, M.N. 1979. Internal migration of people in Nepal. Eastern Anthropology 32:163-176.

Sinclair, A.R.E. 1975. The resource limitation of trophic level in tropical grassland ecosystems. Journal of Animal Ecology 44:497-520.

Smith, J.L.D. and H.R. Mishra. 1992. Status and distribution of Asian elephants in Central Nepal. Oryx 26:34-38.

Sukumar, R. 1989. The Asian Elephant: Ecology and Management. Cambridge University Press, Cambridge, 225 pp.

Thorsrud, K. 1997. Ecological Impacts of Domestic Asian Elephant (Elephus maximus) 
on Vegetation and Wildlife during the Dry Season in Bardia National Park in Lowland Nepal. M.Sc. Thesis in Tropical Ecology, Agriculture University of Norway, Norway.

Wiseman, R., B.R. Page and T.G. Connor. 2004. Woody vegetation change in response to browsing in Ithala Game Reserve, South Africa. South African Journal of Wildlife Research 34:25-37.

Yadav, B.R. 1988. Vegetation Analysis of Lete Panchayat Protected Forest (Mustang). A project paper submitted to T.U., Institute of Forestry Central Campus, Pokhara, Nepal.

Yadav, B.R. 2002. Asian Wild Elephants (Elephas maximus)- People Interface in East Nepal. M.Sc. Thesis, Agricultural University of Norway, Norway 108 pp.

Yadav, B.R. 2005a. Asian wild elephant (Elephas maximus) in eastern Nepal. Nepal Forester's Association, Kathmandu, Nepal. The Journal of Forestry 12(3):68-81.
Yadav, B.R. 2005b. Participatory biodiversity monitoring in buffer zone forest of Royal Bardia National Park. Banko Janakari (A Journal of Forestry Information for Nepal). Department of Forest Research and Survey, Kathmandu Nepal, 115(1):11-18.

Yadav, B.R. and M.K. Chalise. 2013a. Nepalma Jungali Hatti ra Manish Bicha Dwanda (Conflict Between Elephant and Human in Nepal) (in Nepali). $18^{\text {th }}$ Wildlife Week, 2070 BS. DNPWC, Government of Nepal. pp. 1-10.

Yadav, B.R. and M.K. Chalise. 2013b. Study the Exploitation of Natural Resources (Fodder/Grass) in the Habitat of PAs by Domesticated Elephant in Nepal. Report submitted to the Department of National Park and Wildlife Conservation, Kathmandau.

Zobel, D.B., P.K. Jha, M.J. Behan and U.K.R. Yadav. 1987. A Practical Manual for Ecology. Ratna Book Distributors, Kathmandu, Nepal. 